\title{
Embolisation in Epistaxis. Evaluation from 1996 to 2004
}

\author{
Güenzel', T. Roesler², A., Zenev, E. ${ }^{3}$ \\ 'Department of Otolaryngology, Head and Neck Surgery, Frankfurt (Oder), Germany \\ ${ }^{2}$ Department of Radiology, Augsburg, Germany \\ ${ }^{3}$ Department of Otolaryngology, PAN Clinic Köln, Germany
}

\begin{abstract}
:
Objectives: From 1996 to 2004 we investigated 3185 patients suffering from epistaxis/nosebleed: Based on a retrospective analysis, this presentation is going to explain and discuss the benefit of the use of embolisation. Methods: From 1996 to 2004 the team treated 3185 patients with epistaxis; $1.25 \%(40)$ of these patients were treated with embolisation, $0.1 \%$ (3) of them with embolisation of both sides. Embolisation was done distal of maxillary or the sphenopalatine artery with microparticles of polyvinyl alcohol foam (PVA, 150-250 $\mu \mathrm{m}$ ).

Results: In all cases treated with embolisation (40) the severe nosebleed was stopped. Complications, f.i. necrosis of lateral nosewall were not observed.

Conclusions: In case of severe epistaxis embolisation is a gentle and quite reliable therapeutic option with only a few complications. In view of the increasing pressure of cost reduction this method is a possibility of a secure and fast therapy.
\end{abstract}

\section{Objectives}

Treatment of severe epistaxis can encompass many modalities. Rarely, idiopathic or trauma-related nosebleeds can in rare cases be an intractable clinical picture in which normal conservative measures fail. Nasal packing is a frequent procedure to control spontaneous nasal bleeding. Further therapeutic options that can be applied are surgical vascular ligature or selective endovascular occlusion. Extensive collateralization develops after occlusion of the external carotid artery, so the benefits are likely only transient and the procedure cannot be repeated. Endovascular treatment avoids many of the complications associated with surgery and has a lower recurrence rate than the alternative associated with ligation of the internal maxillary artery.

For more than 20 years the endovascular treatment of intractable epistaxis is a routine interventional procedure in our clinic. Around 150 patients have passed the treatment during this time. Transcatheter embolisation is an accepted and effective treatment for intractable epistaxis $[1,3,5,6,9]$.

\section{Methods}

An IRB is not available in our institution, but this is how we obtained proper consent from the patients, in keeping with the mandate of the Declaration of Helsinki.

From 1996 to 2004 we investigated 3185 patients suffering from epistaxis/nosebleed were treated in our ENT-Department (Klinikum Augsburg, Germany) 40 of 
these patients $(1.25 \%)$ by embolisation. Regarding to quality management we carried out a retrospective study to evaluate the therapeutic effectiveness of the super selective embolisation in cases of intractable epistaxis and furthermore to find out the risk of severe complications.

Patients with nosebleed were treated conservatively by means of application locally to bloodbreastfeeding drugs, bipolar koagulation often in combination with nasal packing [5]. With nasal packing a stationary admission occurred. If intractable epistaxis appeared in this stationary stay, the option of the embolisation was discussed interdisciplinary. In heavy cases, bleeding with considerable blood loss, with chronic illnesses (e.g., Morbus Osler) and no contraindications as for example fresh insult was carried out in a contemporary way the embolisation [4]. The patient could be dismissed, when nosebleed was stopped, during the subsequent day.

In the group of 40 patients treated interventionally 8 were female and 32 male, $0.1 \%$ (3) of them with embolisation of both sides. In our neuroradiological interventional management we first perform a super selective diagnostic angiography and second, in regard to the diagnostic result, an embolisation of either the distal maxillary or the sphenopalatine artery, and possibly also the facial artery. The diagnostic procedure was done on the clinically bleeding site with a F5 catheter (Figure 1 and 2).

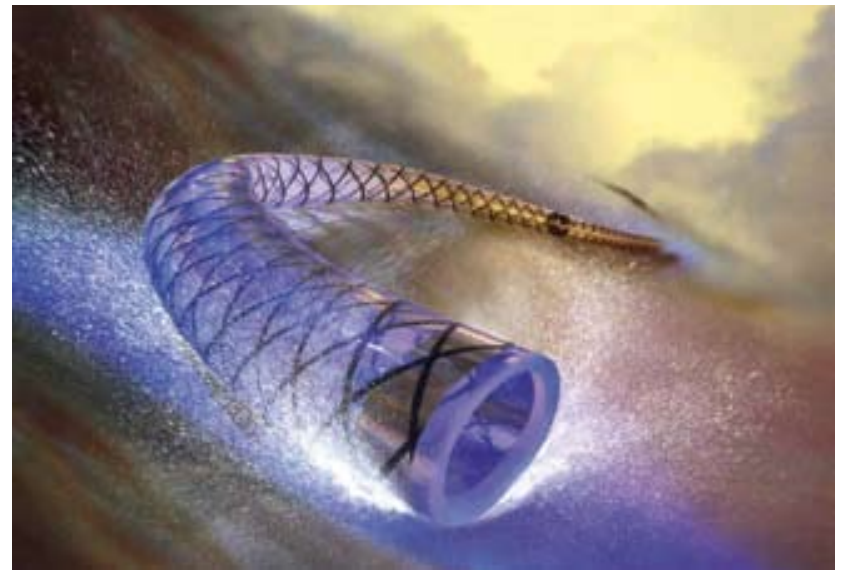

Figure 1: Microcatheter Turbo-Tracker

Leaving this one in the ostium of the external carotid artery, sometimes below the carotid bifurcation as a guiding catheter, we inserted coaxially and advanced a steam shaped micro catheter system

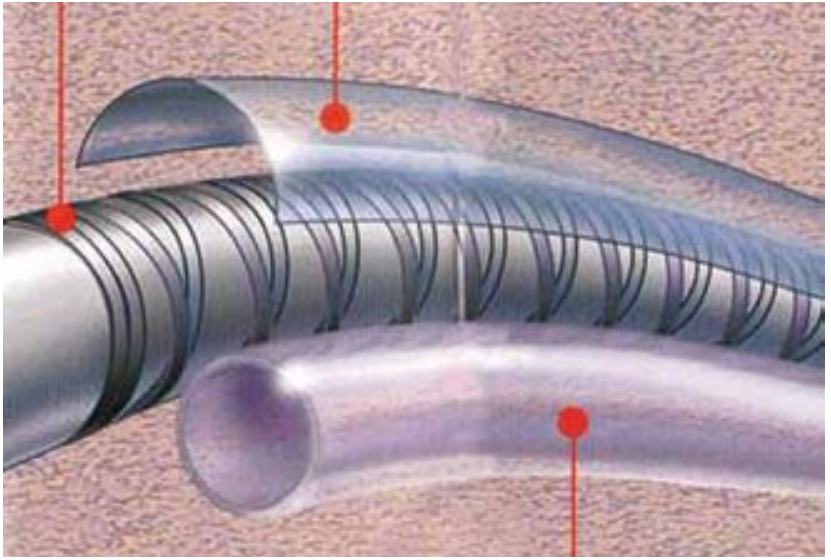

Figure 2: Microcatheter

0.018 Inch guide wire with a $45^{\circ}$ or $90^{\circ}$ tip as far as possible distal in the sphenopalatine portion of the maxillary artery. We always took care of the anastomosis to the ICA (artery of the foramen rotundum) or the ophthalmic artery. Therefore the tip of the micro catheter was always positioned behind this critical point. Figure 3 and 4 show the original microcatheter and set.

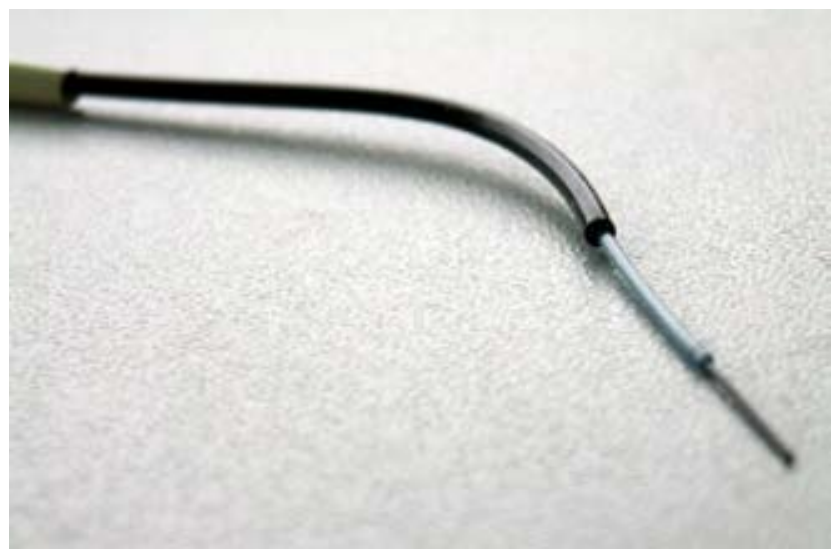

Figure 3: Original microcatheter

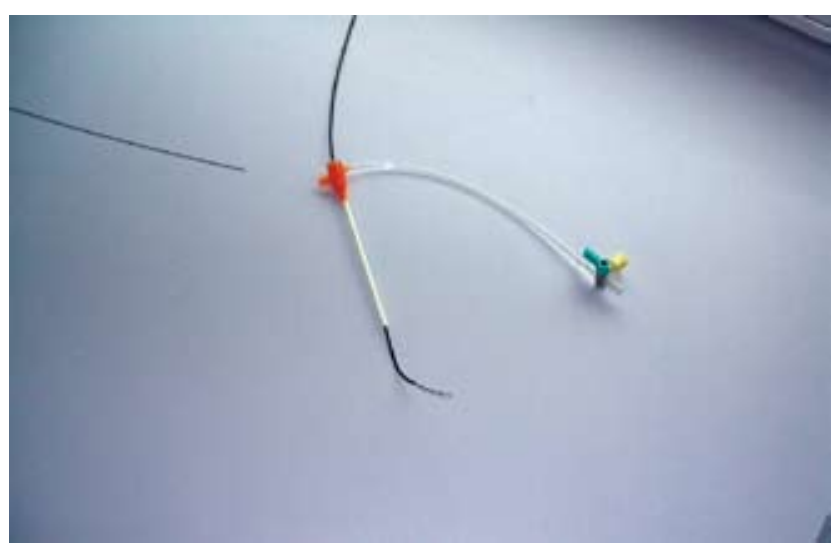

Figure 4: Original set 
Naturally, careful manipulation during the whole procedure is imperative to prevent vasospasm. The microcatheter system in place we performed the embolisation with low pressure injections of PVA micro particles $150-250 \mu \mathrm{m}$ (Figure 5).

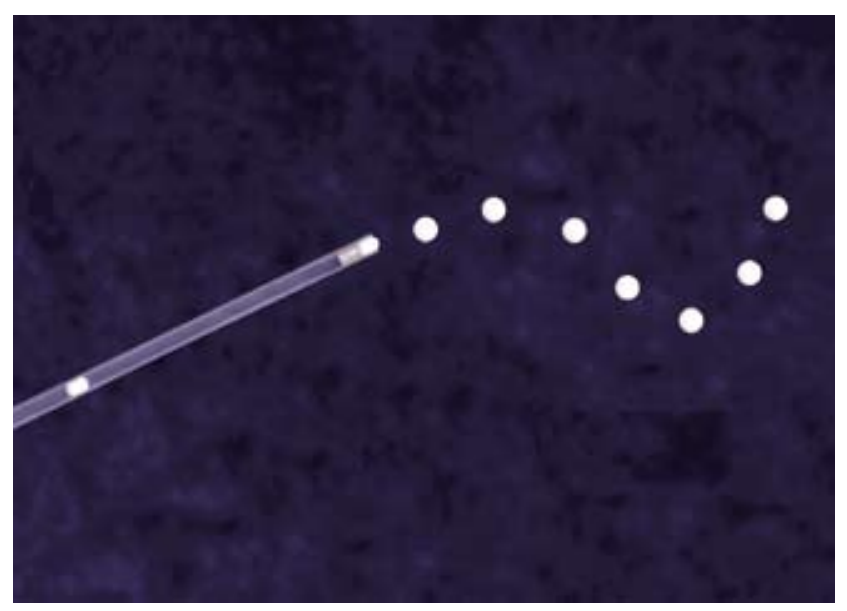

Figure 5: Microcatheter and PVA

The fluoroscopic control is absolutely essential to detect any ophthalmic anastomosis - which cannot be seen in the diagnostic angiography - and to show the flow reduction during the progress of embolisation. If the following control angiography doesn't demonstrate any supply of the bleeding bed from one of the different branches, e.g. the facial artery, the intervention can be finished. Only in cases of bilateral haemorrhage or lack of precise clinical determination of the bleeding source we perform a bilateral angiography and embolisation. This was necessary with 3 patients of our collective.

\section{Results}

The evaluation of angiographic data shows for all 40 patients a technically successful embolisation of the haemorrhage with PVA. In around 50\% of the cases the embolisation took place super selective in the sphenopalatine artery, in $44 \%$ we could only reach the distal maxillary artery without provocation a vasospasm and in $6 \%$ we embolised the bleeding source from the facial artery. The technical problems during the interventional treatment were caused by $10 \%$ by abnormal vessels (variants), by atherosclerotic vessels or by temporary or persistent vasospasm.

In all cases treated with embolisation (40) the severe nosebleed was stopped. Complications, f. i. necrosis of lateral nose wall or hemi paresis were not observed (Figure 6,7).

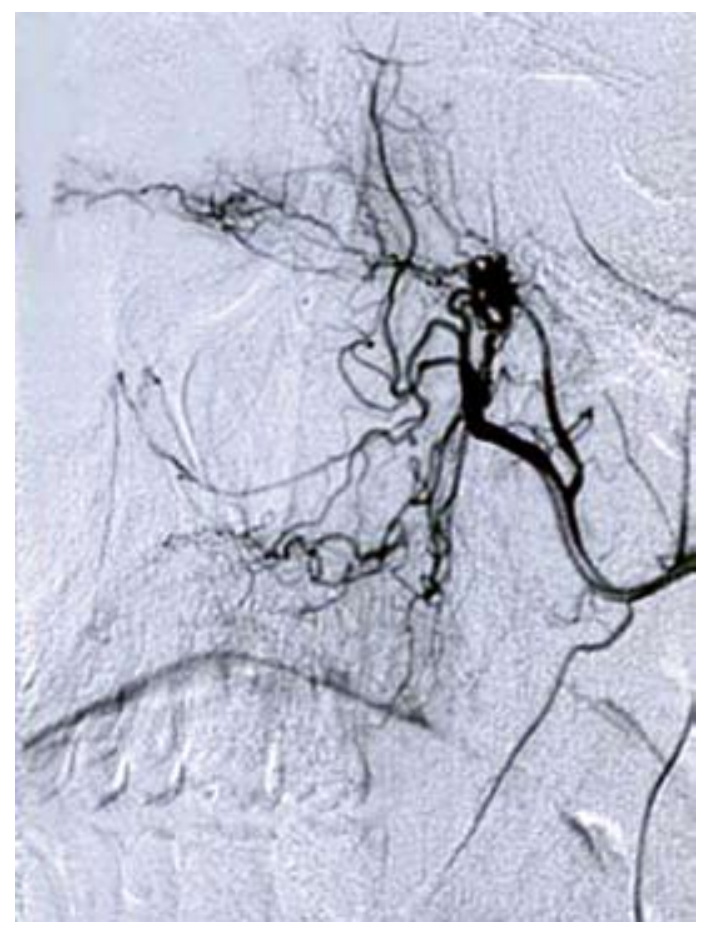

Figure 6: Before embolisation

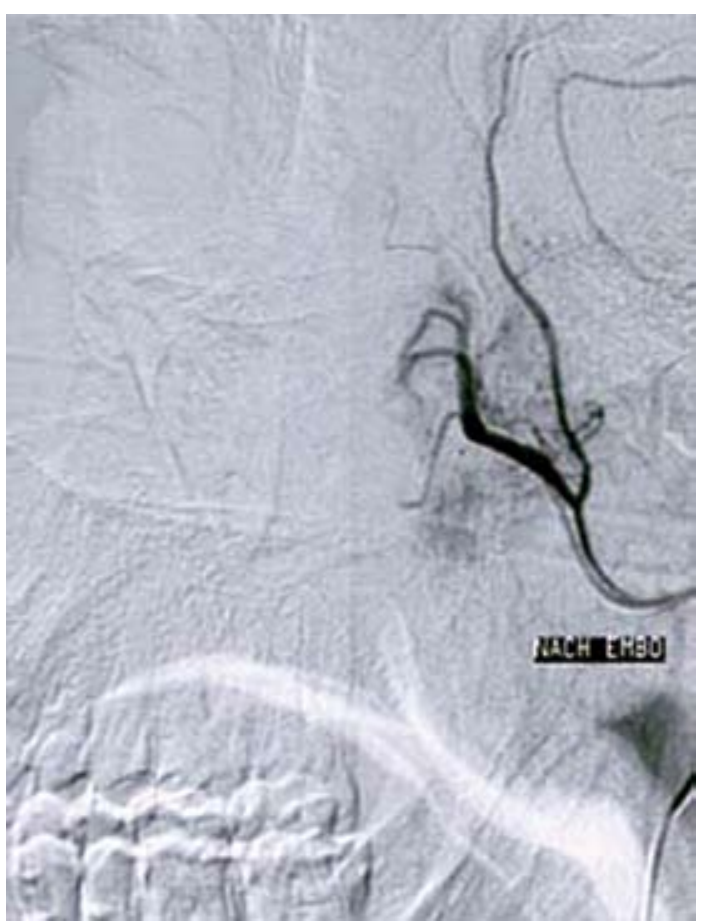

Figure 7: After embolisation

In a other study regarding the interventional therapy from 1990 to 1997 we treated 67 patient with this disease, 47 were male and 20 female. The complication ratio was higher than compared to the actual collective. In the clinical reports (1990-1997) were four cases of hemi paresis. The symptoms of three 
of these patients were completely dissolved after 24 hours without any neurological deficit. One patient, a 82 years old lady, with cardiac problems, stroke of the right MCA and an operation of the left carotid bifurcation suffered acutely from intractable epistaxis and was therefore successfully treated. At the end of the intervention she got a right hemi paresis and didn't recover. The other persistent complication was amaurosis with a 68 years old male. Anamnestically known was a stroke and an externa-interna-bypass over the right superficial temporal artery having a complete ICA-occlusion. The first diagnostic angiography was done from transfemoral and interrupted because the aortic arch couldn't be reached. From the right side via transbrachial an ophthalmic anastomosis was seen, therefore the embolisation could only be performed in the right facial artery. The epistaxis was treated successfully but the ophthalmologist diagnosed an incomplete occlusion of the central retinal artery later.

After our retrospective analysis of these two severe complications and the evaluation of the clinical and angiographic data the endovascular treatment of epistaxis was not the treatment of choice in these patient. For this method it is important to now the risk.

In our latest study (from 1996 -2004) we can show, that there were no major complications.

\section{Reference:}

1. Christensen NP, Smith DS, Barnwell SL et al. Arterial embolization in the management of posterior epistaxis. Otolaryngol Head Neck Surg 2005 Nov; 133(5): 748-53.

2. Pino Rivero V, Gonzalez Palomino A, Trinidad Ruiz G et al. Postraumatic massive epistaxis. An indication for selective arterial embolization An Otorrinolaringol Ibero Am. 2005; 32(5): 453-8.

3. Duncan IC, Fourie PA, le Grange CE et al. Endovascular treatment of intractable epistaxis--results of a 4-year local audit. S Afr Med J. 2004 May; 94(5): 373-8.

4. Elden L, Montanera W, Terbrugge K et al. Angiographic embolization for the treatment of epistaxis: a review of 108 cases. Otolaryngol Head Neck Surg. 1994 Jul; 111(1): 44-50.

5. Evans AS, Young D, Adamson R. Is the nasal tampon a suitable treatment for epistaxis in Accident \& Emergency? A comparison

\section{Conclusions}

In case of severe epistaxis, embolisation is a gentle and quite reliable therapeutic option with only a few complications [6]. In view of the increasing pressure of cost reduction this method is a possibility of a sure and fast therapy [1]. Furthermore, we can cut short the stay in hospital for the patient. This method is not painful and easy to perform for a trained radiological/neuroradiological interventionalist. The super selective embolisation of the distal maxillary or sphenopalatine artery is a successful and elegant method to treat intractable epistaxis with excellent long term results. The patients were also hospitalized for a shorter period than those with only nasal packing. A study from Vergara et al shows the efficiency of surgical versus non-surgical treatment in patients hospitalized for epistaxis regarding the source of bleeding [8]. The patients with super selective embolisation are hispitalized only 2-3 days. The major complication rate of $2.6 \%$ (1990-1997) and 0\% (1996-2004) has to be taken into account.

In conclusion, patients with severe epistaxis should be treated by a multidisciplinary approach. If clinical examination by an ENT surgeon, possibly cautery and endonasal packing is insufficient, angiography and arterial super selective embolisation is the method of choice $[2,7]$.

of outcomes for ENT and A\&E packed patients. J Laryngol Otol. 2004 Jan; 118(1): 12-4.

6. Nickel J, Sachs W, Andresen R. Selective embolisation of posttraumatic intractable epistaxis. Rontgenpraxis. 2003; 55(2): 72-7. German.

7. Mahadevia AA, Murphy KJ, Obray R et al. Embolization for intractable epistaxis. Tech Vasc Interv Radiol. 2005 Sep; 8(3): 134-8.

8. Vergara Hernandez J, Ordonez Ordonez LE. Surgical versus nonsurgical treatment of posterior epistaxis. Acta Otorrinolaringol Esp. 2006 Jan; 57(1): 41-6. Spanish.

9. Zhang P, Chu Y, Luo Y at al. Clinical analysis of selected transarterial embolization in treating uncontrolled epistaxis. Lin Chuang Er Bi Yan Hou Ke Za Zhi. 2003 Nov; 17(11): 657-9. Chinese.

\author{
Corresponding author: \\ Dr. med. Thomas Günzel \\ Head Department of Otolaryngology, Head and Neck Surgery, Klinik für HNO-Heilkunde, Kopf- und Halschirurgie, \\ Plastische Operationen Klinikum Frankfurt (Oder) GmbH \\ Müllroser Chaussee 7, \\ G- 15236 Frankfurt (Oder) \\ Germany \\ Tel.: +49 335548 2680; Fax: +493355482699 \\ E-mail: th.guenzel.ch@klinikumffo.de
}

Oral presentation: at the 2006 Annual Meeting \& OTO EXPO, September 17-20, 2006 